\title{
Obesity and reproduction: a review article
}

\begin{abstract}
Obesity is a worldwide health problem related to many reproductive health issues. It can impair ovarian function, affecting the correct maturation of the oocyte, normal fertilization and embryo quality. Obesity can also affect cellular metabolism by damaging mitochondria. Implantation and endometrial receptivity may be diminished, according to some studies. Correct placentation is affected too, once pregnant, obese women are at a higher risk of obstetric complications. Obstetricians and gynecologists should be aware of the impact of obesity to properly asses their patients. Further studies should focus on a better comprehension of the mechanisms involved in the adverse effects of obesity on reproduction to develop targeted therapies.
\end{abstract}

Volume 10 Issue 2 - 2019

\author{
Quintana Ramiro Hector, Quintana Tomas \\ Manuel,Viale Luz \\ Genesis Biologica, University of Buenos Aires, Argentina
}

Correspondence: Dr. Quintana, Ramiro Hector, Genesis Biologica, University of Buenos Aires Address Marcelo T. de Alvear 2320, Ciudad Autonoma de Buenos Aires, Argentina, Tel 54I|40255772, Email ramiroquintana@hotmail.com

Received: September 27, 2018 | Published: March 14, 2019

\section{Introduction}

Obesity constitutes a worldwide health issue that can impair the functioning of a variety of body functions, originating diseases that can even lead to death.

Reproduction, in both men and women, can also be affected by in different ways in the obese population.

Obesity is most commonly defined by body mass index (BMI), although several other methods are available, BMI has proven the simplest one. The amount and location of fat tissue, especially in the waist area, is also an important factor. A BMI of $\mathrm{R} 30 \mathrm{~kg} / \mathrm{m}^{2}$ is considered to be obesity, with severe and morbid obesity defined as BMIs of 35 to $<40 \mathrm{~kg} / \mathrm{m}^{2}$ and $\mathrm{R} 40 \mathrm{~kg} / \mathrm{m}^{2}$, respectively.

The World Health Organization (WHO) estimates around 600 million obese people in the world. ${ }^{2}$

The impact of obesity has not gone unnoticed; mainly due to the increase risk of obstetric complications in this group. ${ }^{3}$ Around 35\% of maternal mortality may be attributed to complications in obese women.

There are other factors at play; in the US prevalence varies markedly by locale and is higher in black and Hispanic adults and lower in Asiatic adults. ${ }^{2,4}$

We aim to briefly summarize the different ways in which obesity can affect fertility in women, altering the correct functioning of the ovary, embryo quality, endometrial receptivity, implantation and during gestation and labor.

We found that it is also important to properly educate the future parents on their role in the nutrition and health of their children, since obesity can be transmitted from one generation to another. ${ }^{5}$ Following conception, maternal obesity is associated with adiposity in the offspring, generating a self-perpetuating cycle set by the mother.

\section{Obesity and ovarian function}

The adverse effects of obesity on ovulation have been classically observed. This ovarian dysfunction can lead to amenorrhea, as first described by Stein-Leventhal.

In obese woman, all the structures of the ovulatory follicle can be affected. An increase in the concentration of insulin, lactate, triglycerides, $\mathrm{C}$ reactive protein and lectin in the follicular fluid of these women has been found. ${ }^{6}$
It is believed that increased lipotoxicity, a cellular response to a high lipid extracellular environment, is a key factor to ovulatory dysfunction in these patients.

Studies have demonstrated that mouse cumulus oocyte complexes (COCs) exhibit lipotoxicity. Organelles like the endoplasmic reticulum (ER) are particularly affected, where its impaired function disrupts protein secretion pathways and triggers the unfolded protein response (UPR) (Kaufman, 1999; Ozcan and Tabas, 2012). ${ }^{7}$

Abnormalities in the meiotic spindle have also been reported, which can lead to a lower ovulation rate and poor fertilization in these patients.

The mitochondria are the organelles where the energy of the cells is generated, producing adenosine triphosphate (ATP) during oocyte maturation and blastocyst formation. ${ }^{8}$ It has a double membrane and an inner membrane that facilitates the electron transportation. Concentration and location of mitochondria in the cytoplasm dramatically shift during the ovulatory cycle. ${ }^{9}$

Studies performed in mice show that oocytes must have a minimum number of mitochondria (with 40,000 to 50,000 copies of mtDNA) in order produce an embryo that will result in a viable fetus.

Obese patients show certain degree of mitochondrial dysfunction such as alteration the internal membrane, poor electron exchange and variation in the concentration and location of the mitochondria. All this can lead to diminished oocyte quality, fertilization rates, blastocyst production and implantation rates.

\section{Obesity and endometrial receptivity}

There is conflicting data as to whether obesity can affect the implantation rates. ${ }^{1}$ Some studies have shown no difference in the implantation rates compared to control subjects. ${ }^{10,11}$ However, other studies, like the one carried out by Desolle et al found a negative correlation. ${ }^{12}$ On 450 cycles using donor oocytes and frozen embryos found that BMI is an independent predictor of clinical pregnancy. In this study, other factors like maternal age and embryo quality could be excluded.

Furthermore, studies in mice have shown that endometrial decidualization is impaired in obese mice, which can lead to poor implantation rates. ${ }^{13}$

Decidualization defects not only lead to compromised endometrial receptivity and poor implantation, when placentation is affected other complications are seen. Obese women present higher risk of 
pregnancy induced hypertension and stillbirth, complications strongly linked to placental dysfunction. ${ }^{14}$ Proinflammatory cytokines and reactive oxygen species (ROS) induce endothelial dysfunction and lead to abnormal placentation.

\section{Discussion}

Identifying the mechanisms behind the poor reproductive outcome in obese women leads us into a better understanding of a complex disease, with the intention to develop targeted therapies. ${ }^{15}$

Weight loss may seem the cornerstone of treatment in these patients, but other approaches have shown a faster response: changes in lifestyle, incremented physical activity and reduction of mental stress. ${ }^{16}$ Establishing the right moment during weight loss in which to start a fertility treatment has also proven a challenge, considering other variables like maternal age.

A multidisciplinary approach of the obese patient seems to be of utter importance. Professionals should focus on improving the cellular environment for oocyte maturation, embryo development, achieving better implantation rates and preventing future obstetric complications.

\section{Acknowledgments}

None.

\section{Conflicts of interest}

The authors declare no conflicts of interest.

\section{References}

1. Broughton DE, Moley KH. Obesity and female infertility: potential mediators of obesity's impact. Fertil Steril. 2017;107(4):840-847.

2. Luke B. Adverse effects of female obesity and interaction with race on reproductive potential. Fertil Steril. 2017;107(4):868-877.

3. Franco LP, Morais CC, Cominetti C. Normal-weight obesity syndrome: diagnosis, prevalence, and clinical implications. Nutr Rev 2016;74(9):558-570.
4. Centers for disease control and prevention.

5. Ruager-Martin R, Hyde MJ, Modi N. Maternal obesity and infant outcomes. Early Hum Dev. 2010;86(11):715-722.

6. Robker RL, Akison LK, Bennett BD, et al. Obese women exhibit differences in ovarian metabolites, hormones, and gene expression compared with moderate-weight women. J Clin Endocrinol Metab. 2009;94(5):1533-1540.

7. Wu LL, Norman RJ, Robker RL. The impact of obesity on oocytes: evidence for lipotoxicity mechanisms. Reprod Fertil Dev. 2011;24(1):29-34.

8. Van Blerkom J, Davis PW, Lee J. ATP content of human oocytes and developmental potential and outcome after in-vitro fertilization and embryo transfer. Hum Reprod. 1995;10(2):415-424.

9. Dumollard R, Duchen M, Carroll J. The role of mitochondrial function in the oocyte and embryo. Curr Top Dev Biol. 2007;77:21-49.

10. Styne-Gross A, Elkind-Hirsch K, Scott RT. Obesity does not impact implantation rates or pregnancy outcome in women attempting conception through oocyte donation. Fertil Steril. 2005;83(6):16291634.

11. Wattanakumtornkul S, Damario MA, Stevens Hall SA, et al. Body mass index and uterine receptivity in the oocyte donation model. Fertil Steril. 2003;80:336-340.

12. Dessolle L, Darai E, Cornet D, et al. Determinants of pregnancy rate in the donor oocyte model: a multivariate analysis of 450 frozen-thawed embryo transfers. Hum Reprod. 2009;24:3082-3089.

13. Rhee JS, Saben JL, Mayer AL, et al. Dietinducedobesity impairs endometrial stromal cell decidualization: a potential role for impaired autophagy. Hum Reprod. 2016;31:1315-1326.

14. Palomba S, de Wilde MA, Falbo A, et al. Pregnancy complications in women with polycystic ovary syndrome. Hum Reprod Update. 2015;21(5):575-592.

15. Elia EM, Bazzano MV, Quintana R, et al. Reproductive disorders in obesity. Integr Obesity Diabetes. 2015;1(1):20-25.

16. Legro RS. Effects of obesity treatment on female reproduction: results do not match expectations. Fertil Steril. 2017;107(4):860-867. 